\title{
Modal-Expansion Analysis of Multiple Monopole Antennas
}

\author{
Quanxin Wang, Zhongxiang Shen, and Erping Li
}

Received 24 July 2007; Accepted 17 October 2007

Recommended by Joshua Le-Wei Li

The modal-expansion method is employed to analyze an array of multiple monopole antennas. A perfectly conducting plate is introduced at the top of the monopole array to facilitate the modal-expansion analysis. Expansion coefficients in the field expressions are found by enforcing continuity conditions of the tangential field components across the regional surfaces. Cylindrical function's addition theorem is employed to realize the transformation of field expressions in different coordinate systems. Numerical results for the $S$-parameters of a two-monopole antenna are presented and they are in good agreement with experimental ones. Also examined is the effect of the distance between two monopoles on the antenna's mutual coupling and radiation pattern. A fourmonopole antenna is studied for its beam-steering capability and simulated results for its radiation properties are compared with those obtained by high frequency structure simulator (HFSS).

Copyright (c) 2007 Quanxin Wang et al. This is an open access article distributed under the Creative Commons Attribution License, which permits unrestricted use, distribution, and reproduction in any medium, provided the original work is properly cited.

\section{INTRODUCTION}

Monopole antenna arrays fed through an infinite ground plane by coaxial transmission lines are extensively used in various communication systems due to their desirable features, such as geometrical simplicity, low construction cost, polarization purity, and reasonable bandwidth $[1,2]$. Many analysis methods have been proposed to characterize a single-monopole antenna's radiation performance, such as the integral equation method for an electrically thick monopole [3], the singular integral equation formulation [4], and the conservation of complex power technique [5]. Monopole antenna arrays have also been studied by many researchers using different methods, such as the integral equation method for coupled monopoles [6], the method of moments [7], and the radial guide field matching method (RGFMM) [8]. Tomasic and Hessel studied the problem of a linear array of coaxially fed monopoles radiating into a parallel-plate region [2]. Researchers at ATR Adaptive Communications Research Lab have done much work for electronically steerable passive array radiators (ESPAR) using monopole antennas [9-11].

The modal-expansion method, originally presented by Schwering [12] and Morgan [13, 14], is computationally very efficient and has been employed to analyze various monopole problems by Shen and MacPhie $[15,16]$, Bialkowski $[17,18]$, and Chen et al. [19]. This paper applies the modal-expansion method to study the radiation characteristics of an array of multiple monopole antennas. The motivation of our study is to understand the mutual coupling effect between multi- ple monopoles over an infinite ground plane and to design beam-steerable monopole arrays. The problem of multiple symmetric dipole antennas is basically equivalent to the one considered in this paper, according to the image theory. Antenna arrays capable of beam steering are very useful in various communication systems, such as the global system for mobile communications, radio frequency identification, and wireless positioning systems.

As in $[12,14]$, an infinite perfectly conducting boundary is introduced at the top of the monopole array to confine the domain of interest so that discrete modal-expansion functions can be used to represent radiated electromagnetic fields. The introduced conducting boundary has no significant effect on the analysis of the monopole array because the main radiation from the antenna array is in the horizontal direction and the radiation is null in the vertical direction. Cylindrical function's addition theorem is employed to realize the coordinate transformation between different monopoles. Due to the fact that the coaxial feed line for monopole antennas can be equivalently represented by a small gap feed when the dimensional parameters of the coaxial feed line are electrically small [17], in this paper, we use small gap sources, rather than the practical coaxial feeds $[15,16]$, to simplify the mathematical formulation and numerical computation.

It has been demonstrated in $[12,14,15]$ that the modalexpansion method is numerically accurate and yet computationally efficient for modeling monopole antennas. Compared to the method of moments, the modal-expansion method can be directly applied to thick monopoles without 


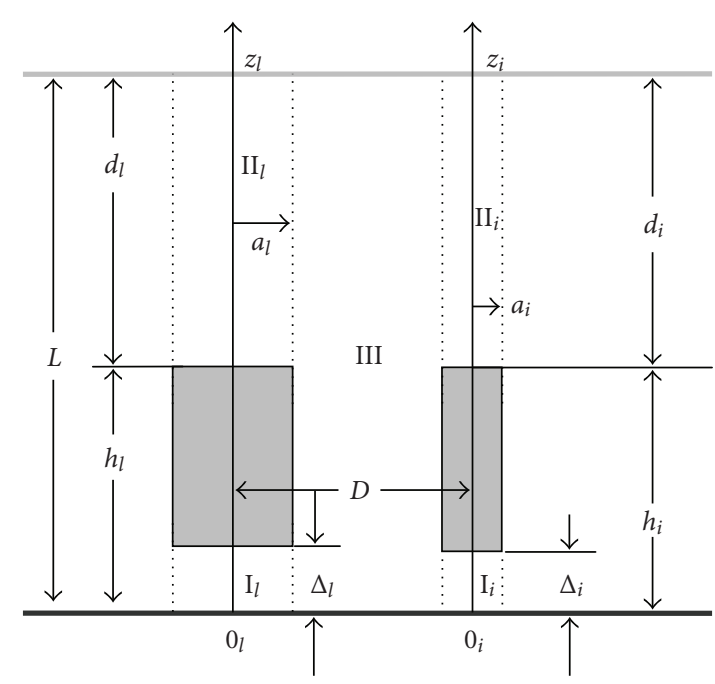

(a) Side view

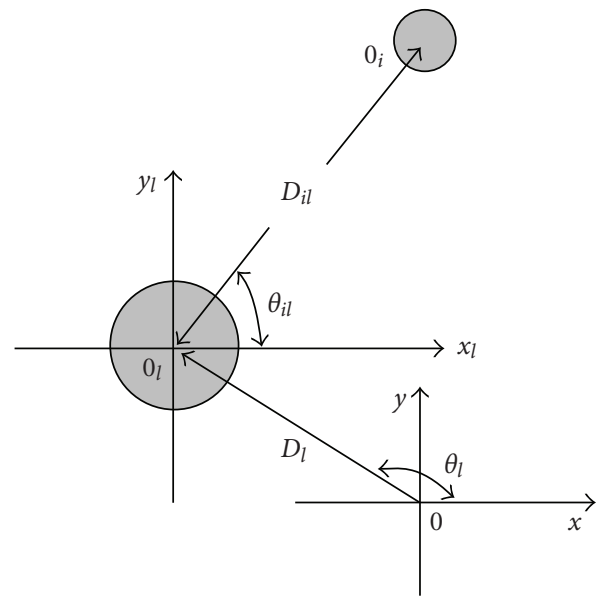

(b) Top view

FIGURE 1: Analysis model of an array of multiple monopole antennas.

invoking the thick-wire kernel. Different from the linear array of monopoles in [1] and the circular array in [7], this paper focuses on the analysis of an array of multiple monopoles, which can have different radii and lengths at arbitrary locations in the ground plane.

The remaining of the paper is organized as follows. In Section 2, the detailed formulation for the radiation problem of an array of multiple monopoles using the modalexpansion method is presented. A planar conducting boundary is introduced at the top of the monopole array to confine the domain of interest. Section 3 provides numerical results for a two-monopole antenna's $S$-parameters and radiation patterns. A comparison between our numerical results with experimental ones is made and a good agreement between them is observed. A four-monopole antenna that is capable of beam-steering is studied and its radiation characteristics are examined with simulated results presented. Some concluding remarks are given in Section 4.

\section{FORMULATION}

The geometry of an array of $P$ monopole antennas fed by gap sources over an infinite ground plane is shown in Figure 1. It is seen that all the cylindrical coordinate systems have the same $x-y$ plane (ground plane) and different origins are employed to facilitate the analysis of the antenna structure. Monopole $i(i=1,2, \ldots, P)$ is of radius $a_{i}$ and has a height of $h_{i}-\Delta_{i}$ with $\Delta_{i}$ being the gap, where a voltage source $V_{i}$ is applied as the excitation along the finite gap between monopole $i$ and the ground plane. A perfectly conducting boundary is introduced at the top of the monopole array to facilitate the modal-expansion analysis $[14,15]$.

As shown in Figure 1, the whole structure between the ground plane and the upper conducting boundary can be divided into a number of Regions $\mathrm{I}_{1}, \mathrm{II}_{1}, \mathrm{I}_{2}, \mathrm{II}_{2}, \ldots, \mathrm{I}_{P}, \mathrm{II}_{P}$ and
III. A uniform electric field is assumed across the gap Regions $\mathrm{I}_{i}$ between monopole $i$ and the ground plane. The electromagnetic fields in Regions $\mathrm{II}_{i}$ and III can be expressed as the summation of their modal functions weighted with expansion coefficients to be determined.

It is obvious that the fields radiated by an individual monopole in the absence of other elements are axially symmetric with respect to its axis. Therefore, the radiated fields from monopole $i$ in the array configuration are $\phi_{i}$-invariant if no multiple reflections between monopoles are considered. Nevertheless, due to the mutual coupling between monopoles, there should be $\phi_{i}$-variation for the electromagnetic fields in Regions $\mathrm{II}_{i}$ when monopole $i$ is in the array configuration. Because the radii of monopole antennas are usually small compared to the free-space wavelength and when the monopoles are not too close to each other, the $\phi_{i}$ variation for the fields in Regions $\mathrm{II}_{i}$, due to the mutual coupling between monopole antennas, is very small. Therefore, we can neglect the $\phi_{i}$-variation for the fields in Regions $\mathrm{II}_{i}$ to reduce the complexity in derivation and computation. With this approximation of ignoring multiple reflections between monopoles, the relevant electric and magnetic field components due to gap source $V_{i}$ in Regions $\mathrm{I}_{i}, \mathrm{II}_{i}$, and III can be expressed as follows.

In Regions $\mathrm{I}_{i},\left(\rho_{i} \leq a_{i}, 0 \leq z \leq \Delta_{i}, i=1,2, \ldots, P\right)$ we know that

$$
E_{z}^{\mathrm{I}_{i}}=\frac{V_{i}}{\Delta_{i}}
$$
have

In Regions $\mathrm{II}_{i},\left(\rho_{i} \leq a_{i}, h_{i} \leq z \leq L, i=1,2, \ldots, P\right)$ we

$$
E_{z}^{\mathrm{II} i}=\frac{1}{j \omega \varepsilon_{0}} \sum_{n=0}^{N_{2}^{i}} B_{i n} \gamma_{n}^{\mathrm{II}_{i}} J_{0}\left(\gamma_{n}^{\mathrm{II}_{i}} \rho_{i}\right) \cos \left[\frac{n \pi\left(z-h_{i}\right)}{d_{i}}\right]
$$




$$
H_{\phi}^{\mathrm{II}_{i}}=\sum_{n=0}^{N_{2}^{i}} B_{i n} J_{1}\left(\gamma_{n}^{\mathrm{II}_{i}} \rho_{i}\right) \cos \left[\frac{n \pi\left(z-h_{i}\right)}{d_{i}}\right],
$$

where $J_{0}$ and $J_{1}$ are the first-kind Bessel functions of order 0 and order 1 , respectively, $B_{\text {in }}$ is the expansion coefficient to be determined, and $\left(\gamma_{n}^{\mathrm{II}_{i}}\right)^{2}=k_{0}^{2}-\left(n \pi / d_{i}\right)^{2}$ with $k_{0}^{2}=\omega^{2} \mu_{0} \varepsilon_{0}$.

In Region III, $\left(\rho_{i} \geq a_{i}, 0 \leq z \leq L, i=1,2, \ldots, P\right)$ under the same condition that the monopole radii are electrically small and these monopoles are not very close to each other, we can assume that the current distribution along all the monopoles' surfaces is axially symmetric. Thus the fields radiated from monopole $i$ can be expressed as

$$
\begin{aligned}
E_{z}^{i} & =\frac{1}{j \omega \varepsilon_{0}} \sum_{n=0}^{N_{1}} A_{i n} \gamma_{n} H_{0}^{(2)}\left(\gamma_{n} \rho_{i}\right) \cos \left(\frac{n \pi z}{L}\right), \\
H_{\phi}^{i} & =\sum_{n=0}^{N_{1}} A_{i n} H_{1}^{(2)}\left(\gamma_{n} \rho_{i}\right) \cos \left(\frac{n \pi z}{L}\right),
\end{aligned}
$$

where $H_{0}^{(2)}$ and $H_{1}^{(2)}$ are the second-kind Hankel functions of order 0 and order 1 , respectively, $A_{\text {in }}$ is the expansion coefficient to be determined, and $\gamma_{n}^{2}=k_{0}^{2}-(n \pi / L)^{2}$.

The total electric field in Region III can then be expressed as the summation of the electric fields radiated from all the monopoles

$$
E_{z}=\sum_{i=1}^{P} E_{z}^{i}=\frac{1}{j \omega \varepsilon_{0}} \sum_{i=1}^{P} \sum_{n=0}^{N_{1}} A_{i n} \gamma_{n} H_{0}^{(2)}\left(\gamma_{n} \rho_{i}\right) \cos \left(\frac{n \pi z}{L}\right)
$$

Using the addition theorem for cylindrical functions [20], the total electric field radiated from the monopoles in Region III can be expressed in one coordinate system (say $x_{i}$ $\left.y_{i}-z_{i}\right)$. Invoking the earlier assumption that the radii of the monopoles are electrically small, only the terms with no $\phi_{i}{ }^{-}$ variation need to be considered when applying the addition theorem at the surface of the monopole $\left(\rho_{i}=a_{i}\right)$. Under this assumption, we have the following approximate expression:

$$
H_{0}^{(2)}\left(\gamma_{n} \rho_{l}\right) \approx H_{0}^{(2)}\left(\gamma_{n} D_{i l}\right) J_{0}\left(\gamma_{n} \rho_{i}\right)
$$

where $D_{i l}$ is the distance from origin $0_{l}$ to origin $0_{i}$ of the coordinate system $x_{i}-y_{i}-z_{i}$. Using (5), (4) can be rewritten in the coordinate system $x_{i}-y_{i}-z_{i}$ as

$$
\begin{aligned}
E_{z}^{\text {total }}\left(\rho_{i}, \phi_{i}\right) & \approx \frac{1}{j \omega \varepsilon_{0}} \sum_{n=0}^{N_{1}} \gamma_{n} \cos \left(\frac{n \pi z}{L}\right) \\
& \times\left[A_{i n} H_{0}^{(2)}\left(\gamma_{n} \rho_{i}\right)+\sum_{\substack{l=1 \\
l \neq i}}^{P} A_{l n} H_{0}^{(2)}\left(\gamma_{n} D_{i l}\right) J_{0}\left(\gamma_{n} \rho_{i}\right)\right] .
\end{aligned}
$$

Based on Maxwell's equations, the magnetic field in Region III can then be obtained from (6) and expressed in the coordinate system $x_{i}-y_{i}-z_{i}$ as

$$
\begin{aligned}
H_{\phi}^{\text {total }}\left(\rho_{i}, \phi_{i}\right) & \approx \sum_{n=0}^{N_{1}} \cos \left(\frac{n \pi z}{L}\right) \\
& \times\left[A_{\text {in }} H_{1}^{(2)}\left(\gamma_{n} \rho_{i}\right)+\sum_{\substack{l=1 \\
l \neq i \\
l \neq i}}^{P} A_{l n} H_{0}^{(2)}\left(\gamma_{n} D_{i l}\right) J_{1}\left(\gamma_{n} \rho_{i}\right)\right] .
\end{aligned}
$$

By applying the boundary conditions that tangential field components must be continuous at $\rho_{i}=a_{i}$, we can obtain that

$$
\begin{aligned}
& E_{z}^{\text {total }}\left(a_{i}, \phi_{i}\right)= \begin{cases}V_{i} / \Delta_{i}, & 0 \leq z \leq \Delta_{i}, \\
0, & \Delta_{i} \leq z \leq h_{i}, \\
E_{z}^{\mathrm{II}_{i}}, & h_{i} \leq z \leq L,\end{cases} \\
& H_{\phi}^{\text {total }}\left(a_{i}, \phi_{i}\right)=H_{\phi}^{\mathrm{II}_{i}}, \quad h_{i} \leq z \leq L,(i=1,2, \ldots, P)
\end{aligned}
$$

From $(8 \mathrm{~b})$ and carrying out the integral $\int_{h_{i}}^{L}(8 \mathrm{~b}) \cdot \cos (n \pi(z-$ $\left.\left.h_{i}\right) / d_{i}\right) d z$, one can explicitly derive $B_{\text {in }}$ as follows:

$$
\begin{aligned}
B_{\text {in }}= & \frac{\varepsilon_{n}}{d_{i} J_{1}\left(\gamma_{n}^{\mathrm{II} i} a_{i}\right)} \\
& \times \sum_{k=0}^{N_{1}}\left[A_{i k} H_{1}^{(2)}\left(\gamma_{k} a_{i}\right)+\sum_{\substack{t=1 \\
t \neq i}}^{P} A_{t k} H_{0}^{(2)}\left(\gamma_{k} D_{i t}\right) J_{1}\left(\gamma_{k} a_{i}\right)\right] F_{n k}^{i} .
\end{aligned}
$$

Substituting $B_{\text {in }}$ in (9) into (8a) and working out the integral $\int_{0}^{L}(8 \mathrm{a}) \cdot \cos (m \pi z / L) d z$, one can obtain the following linear equation system for expansion coefficient $A_{l k}[15,16]$ :

$$
\sum_{l=1}^{P} \sum_{k=0}^{N_{1}} c_{m k}^{i l} A_{l k}=Q_{m}^{i}
$$

After solving (10) we can determine the expansion coefficients $A_{l k}$ [15]. The number of equations in (10) is related to the number of monopoles and the number of modes considered in Region III. The number $N_{1}$ of modes retained is, in turn, dependent upon the distance between the ground plane and the fictitious conducting boundary. The larger the distance is, the more is the number of equations.

Once the coefficient $A_{\text {in }}$ is known, we can find the current distribution along the surface of monopole $i$ from (7):

$$
\begin{aligned}
\mathrm{I}_{i}(z)= & 2 \pi a_{i} \sum_{n=0}^{N_{1}} \cos \left(\frac{n \pi z}{L}\right) \\
& \times\left[A_{i n} H_{1}^{(2)}\left(\gamma_{n} a_{i}\right)+\sum_{\substack{l=1 \\
l \neq i}}^{P} A_{l n} H_{0}^{(2)}\left(\gamma_{n} D_{i l}\right) J_{1}\left(\gamma_{n} a_{i}\right)\right]
\end{aligned}
$$


The S-parameter of the array of multiple monopole antennas can then be found from

$$
[S]=\left([1]+\left[\frac{1}{\sqrt{Y_{c}}}\right][Y]\left[\frac{1}{\sqrt{Y_{c}}}\right]\right)^{-1}\left([1]-\left[\frac{1}{\sqrt{Y_{c}}}\right][Y]\left[\frac{1}{\sqrt{Y_{c}}}\right]\right)
$$

where the ith element $Y_{c i}$ in the diagonal matrix $\left[1 / \sqrt{Y_{c}}\right]$ is the characteristic conductance of the feed line of monopole $i$. In this paper, $Y_{c i}=0.02 S\left(Z_{c i}=50 \mathrm{ohms}\right)$ is chosen as the characteristic conductance of the coaxial feed line. The elements of matrix $[Y]$ can be derived by

$$
\begin{aligned}
Y_{i l}= & \left.\frac{I_{l}\left(\Delta_{l}\right)}{V_{i}}\right|_{V_{l}=0,(l \neq i)}=\frac{2 \pi a_{l}}{V_{i}} \sum_{n=0}^{N_{1}} \cos \left(\frac{n \pi \Delta_{l}}{L}\right) \\
& \times\left[A_{l n} H_{1}^{(2)}\left(\gamma_{n} a_{l}\right)+\sum_{\substack{t=1 \\
t \neq l}}^{P} A_{t n} H_{0}^{(2)}\left(\gamma_{n} D_{l t}\right) J_{1}\left(\gamma_{n} a_{l}\right)\right]_{V_{l}=0,(l \neq i)},
\end{aligned}
$$

where $i, l=1,2, \ldots, P$. It should be mentioned that although the parameter $Y_{i l}$ is obtained from (13) by assuming the given excitation voltage $V_{i}$, the obtained $Y$-parameters and resultant $S$-parameters are independent of the amplitude of excitations.

The mutual coupling between monopole antennas is represented by the transmission coefficients $S_{i j}(i \neq j)$ in (12) or the mutual admittance $Y_{i l}(i \neq l)$ in (13). For the case of two monopoles, the array is considered to be a two-port network

$$
\begin{aligned}
& \mathrm{I}_{1}=Y_{11} V_{1}+Y_{12} V_{2}, \\
& \mathrm{I}_{2}=Y_{21} V_{1}+Y_{22} V_{2} .
\end{aligned}
$$

Due to the mutual coupling between monopole elements, the input conductance $Y_{11}\left(Y_{22}\right)$ is different from $Y_{s}$, which is the admittance in absence of the other monopole. It will be shown later that the distance between two monopoles is the critical parameter affecting the mutual coupling.

Once the electric current distribution along the monopole surface in (11) is obtained, one can calculate the far-field radiation pattern of the array of multiple monopole antennas. The image theory is employed to account for the reflection from the infinite ground plane. In the spherical coordinate system $(r, \theta, \phi)$, the far-zone electric field has only $\theta$-component. Therefore, the far-zone-radiated electric field can be calculated from the following formula:

$$
\begin{aligned}
& E_{\theta}=\frac{j \omega \mu_{0} \sin \theta}{2 \pi} \sum_{i=1}^{P} a_{i} \frac{e^{-j k r_{i}}}{r_{i}} \\
& \times\left[\left.\int_{\Delta_{i}}^{h_{i}} \int_{0}^{2 \pi} H_{\phi_{i}}\right|_{\rho_{i}=a_{i}} \cos \left(k z_{i} \cos \theta\right) e^{j k a_{i} \cos \left(\phi_{i}-\phi\right) \sin \theta} d \phi_{i} d z_{i}\right],
\end{aligned}
$$

where $r_{i}$ is the distance from the field point to $O_{i}$. Substituting (6) in (13) and performing integration over all the

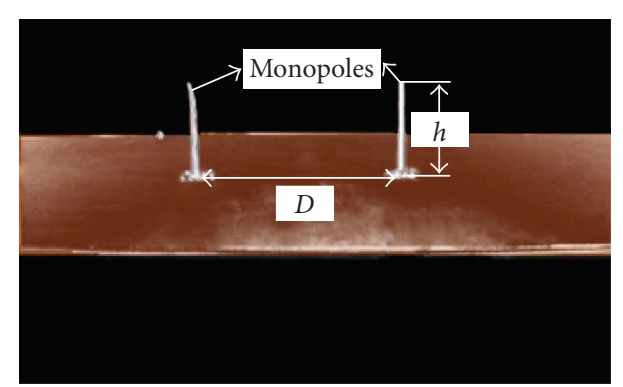

Figure 2: A two-monopole antenna.

monopole surfaces, we have

$$
E_{\theta}=\frac{j \omega \mu_{0} \sin \theta e^{-j k r}}{r} \sum_{i=1}^{p} T_{i} a_{i} J_{0}\left(k a_{i} \sin \theta\right) e^{j k D_{i} \cos \left(\theta_{i}-\phi\right) \sin \theta}
$$

where $\left(D_{i}, \theta_{i}\right)$ is the coordinates of origin $0_{i}$ at the $x-y$ plane (see Figure 1),

$$
\begin{aligned}
& T_{i}=\sum_{n=0}^{N_{1}} s_{i n}(\theta)\left[A_{\text {in }} H_{1}^{(2)}\left(\gamma_{n} a_{i}\right)+\sum_{\substack{l=1 \\
l \neq i}}^{P} A_{l n} H_{0}^{(2)}\left(\gamma_{n} D_{i l}\right) J_{1}\left(\gamma_{n} a_{i}\right)\right] \\
& S_{i n}(\theta)=\int_{\Delta_{i}}^{h_{i}} \cos \frac{n \pi z^{\prime}}{L} \cos \left(k z^{\prime} \cos \theta\right) d z^{\prime} .
\end{aligned}
$$

\section{NUMERICAL RESULTS}

In order to verify the formulation derived and to justify the assumptions made, an experimental model for a twomonopole antenna is constructed, as shown in Figure 2, and its $S$-parameters are measured using a vector network analyzer HP8503A in an anechoic chamber. The dimensional parameters of the constructed two-monopole model are given as follows: radius $a_{1}=a_{2}=2.5 \mathrm{~mm}, h_{1}=h_{2}=h=$ $75.32 \mathrm{~mm}$. The spacing of the ground plane and the upper conducting plate used in our simulation is $L=405 \mathrm{~mm}$, which is more than one free-space wavelength over the frequency range of interest $[15,16]$. In the theoretical analysis, the ground plane and the introduced upper conducting plate are assumed to be of infinite extent, while a finite ground plane of size $120 \mathrm{~cm} \times 120 \mathrm{~cm}$ is employed and no top conducting plate is present in the experiment. The ground plane size used in our experiment is large enough for minimizing the diffraction from the edge [21].

Figure 3 shows the simulated and measured $S$-parameter results of the two-monopole antenna. It can be seen that our calculated results agree very well with measured ones, which verifies the validity of our described formulation and the developed program.

For a two-monopole antenna, one can determine the selfimpedance of one element by assuming that the excitation of the other element is zero. Tables 1 and 2 examine the convergence behavior of the monopole antenna's self-impedance results with respect to the truncation numbers $N_{1}$ and $N_{2}$ for 


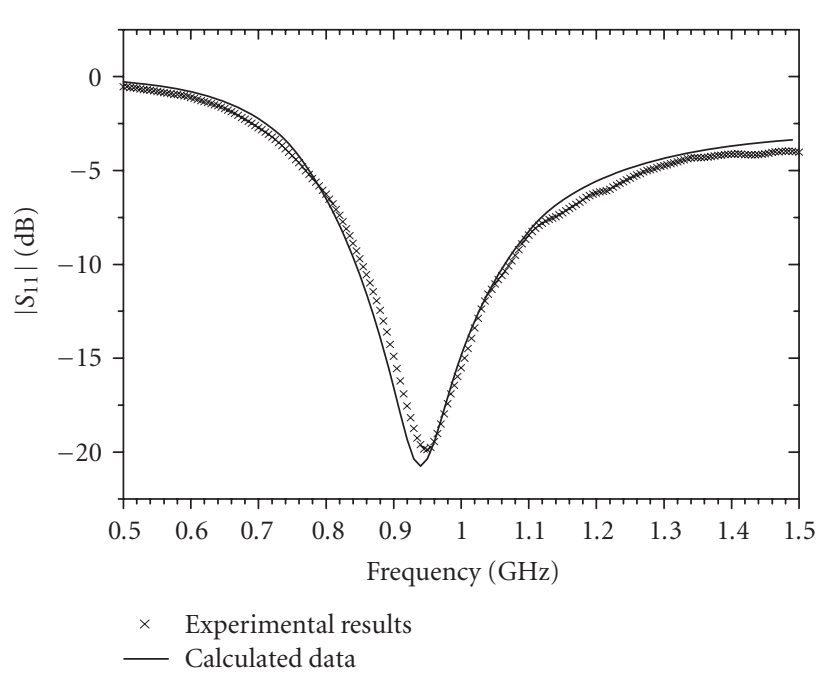

(a) $\left|S_{11}\right|$

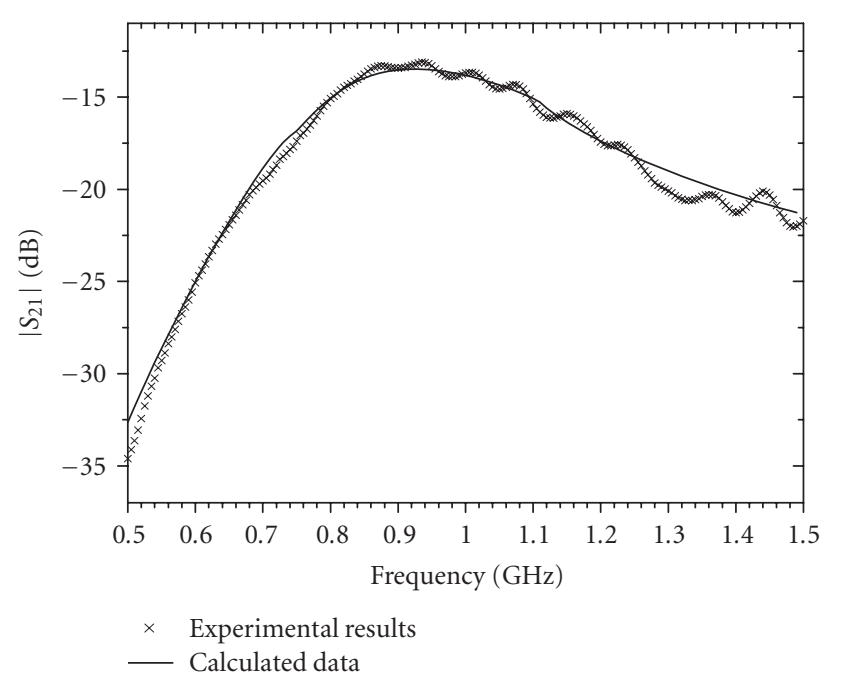

(b) $\left|S_{21}\right|$

FIGURE 3: Simulated and measured $S$-parameters of the two-monopole antenna $(D=160 \mathrm{~mm})$.

TABLE 1: Convergence behavior of the self-impedance result of the monopole antenna with respect to $N_{1}$, the truncation number of the modal expansion in Region III $\left(f=0.9375 \mathrm{GHz}, N_{2}=80\right)$.

\begin{tabular}{lllll}
\hline \multirow{2}{*}{$N_{1}$} & \multicolumn{2}{c}{$D=80 \mathrm{~mm}$} & \multicolumn{2}{c}{$D=160 \mathrm{~mm}$} \\
& $R(\Omega)$ & $X(\Omega)$ & $R(\Omega)$ & $X(\Omega)$ \\
\hline 20 & 32.40 & 16.13 & 38.64 & -6.27 \\
40 & 41.74 & 23.97 & 41.52 & -1.54 \\
80 & 45.89 & 25.84 & 42.69 & -0.39 \\
120 & 47.48 & 26.30 & 43.05 & -0.08 \\
\hline
\end{tabular}

the expansion expressions of the field components in Region III and Regions II $_{i}$ (see Figure 1), respectively. It is seen from Table 1 that the monopole's self-impedance result does not change much when $N_{1}$ increases from 80 to 120. Similarly, it is noticed from Table 2 that $N_{2}=80$ will be good enough to ensure convergent results. In fact, the value of the truncation number $N_{1}$ is related to the spacing between the top conducting plate and the ground plane. The bigger the spacing between these two planes is, the larger is the number of $N_{1}$ required. Similarly, the value of $N_{2}$ is related to the distance between the top ends of the monopoles to the upper plate. In this two-monopole antenna, the two monopoles have the same distance to the upper plate. We can therefore use the same truncation number $N_{2 i}=N_{2}(\mathrm{i}=1,2)$ for the expansions in Regions $\mathrm{II}_{1}$ and $\mathrm{II}_{2}$.

Table 3 examines the effect of the introduced upper conducting boundary on the monopole antenna's selfimpedance. It is seen that the distance from the upper conducting boundary to the monopole's top end has little effect on the self-impedance of the monopole antenna when $d \geq \lambda_{0}$, which validates our assertion that the introduction of the conducting plate has no significant effect on the analysis of multiple-monopole antennas.

In order to investigate the effect of the distance between monopoles on the antenna's performance, the same two-
TABLE 2: Convergence behavior of the self-impedance result of the monopole antenna with respect to $N_{2}$, the truncation number of the modal expansion in Regions $\mathrm{II}_{i}\left(f=0.9375 \mathrm{GHz}, N_{1}=80\right)$.

\begin{tabular}{llllc}
\hline \multirow{2}{*}{$N_{2}$} & \multicolumn{2}{c}{$D=80 \mathrm{~mm}$} & \multicolumn{2}{c}{$D=160 \mathrm{~mm}$} \\
& $R(\Omega)$ & $X(\Omega)$ & $R(\Omega)$ & $X(\Omega)$ \\
\hline 20 & 50.96 & 30.09 & 44.55 & 3.84 \\
40 & 47.89 & 27.54 & 43.38 & 1.26 \\
80 & 45.89 & 25.84 & 42.69 & -0.39 \\
100 & 45.64 & 25.62 & 42.60 & -0.61 \\
\hline
\end{tabular}

TABLe 3: Effect of the distance from the introduced conducting boundary to the monopoles' top end on the monopole antenna's self-impedance $Z=R+X(f=0.9375 \mathrm{GHz})$.

\begin{tabular}{llrlc}
\hline \multirow{2}{*}{$d / \lambda_{0}$} & \multicolumn{2}{c}{$D=80 \mathrm{~mm}$} & \multicolumn{2}{c}{$D=160 \mathrm{~mm}$} \\
& $R(\Omega)$ & $X(\Omega)$ & $R(\Omega)$ & $X(\Omega)$ \\
\hline 0.25 & 43.22 & 17.89 & 31.20 & -1.10 \\
0.50 & 47.47 & 26.76 & 44.74 & -0.79 \\
0.75 & 45.59 & 23.95 & 39.13 & -1.11 \\
1.00 & 45.72 & 25.92 & 42.68 & -0.08 \\
1.50 & 46.50 & 26.50 & 42.53 & 0.52 \\
\hline
\end{tabular}

monopole antenna with a separation of $D=320.0 \mathrm{~mm}$ is considered. Figure 4 shows the simulated and measured $S$-parameter results when the distance between the two monopoles is $D=320.0 \mathrm{~mm}$. A good agreement between simulated and measured results can also be seen in Figure 4. It is noted that the mutual coupling for $D=320.0 \mathrm{~mm}$ is weaker (about $5 \mathrm{~dB}$ less) than that for $D=160 \mathrm{~mm}$, which is expected.

Figure 5 gives the comparison of our computed results for the two-monopole antenna's radiation pattern with those obtained by Ansoft's high frequency structure simulator (HFSS). We can see that our modal-expansion results agree 


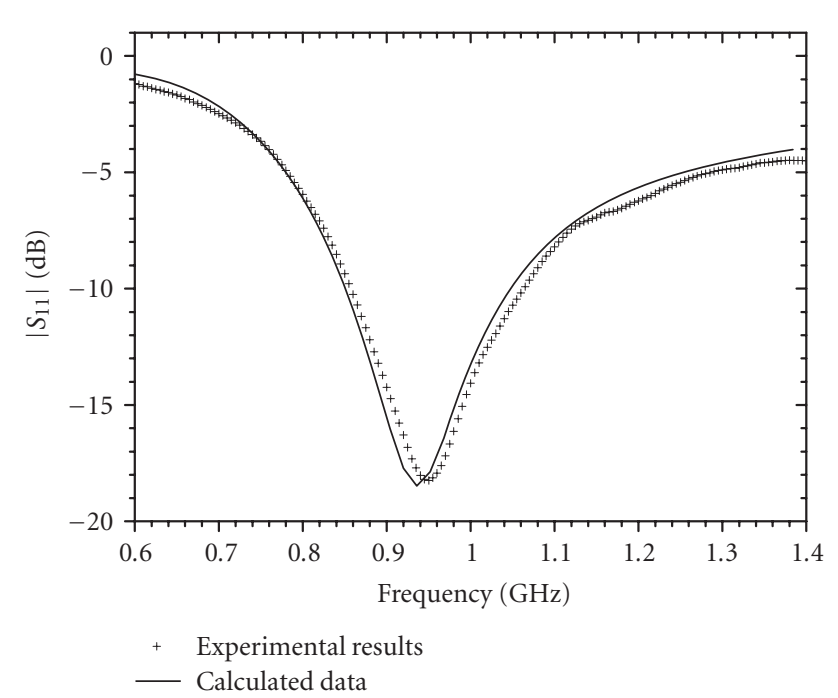

(a) $\left|S_{11}\right|$

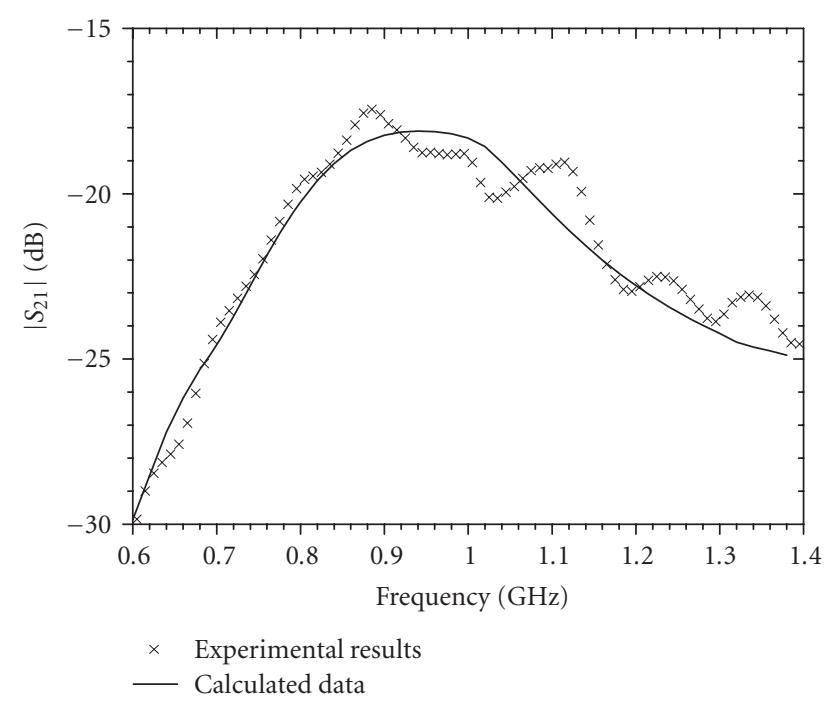

(b) $\left|S_{21}\right|$

FIGURE 4: Simulated and measured $S$-parameters of the two-monopole antenna $(D=320 \mathrm{~mm})$.

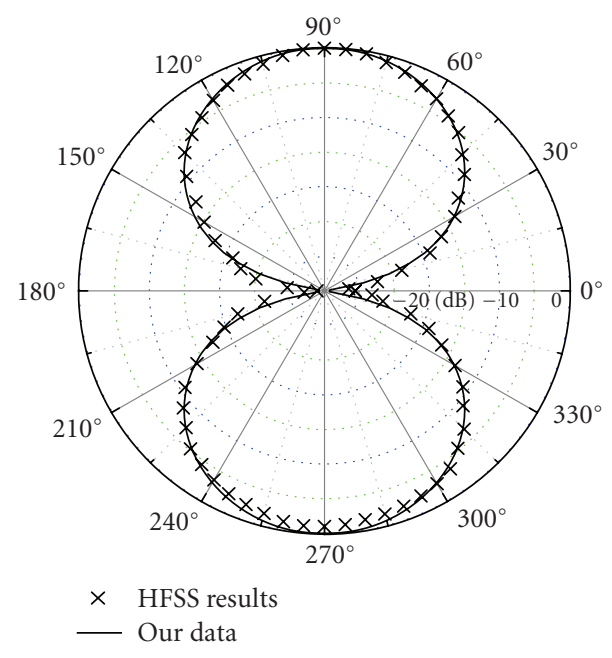

(a) $D=160 \mathrm{~mm}$

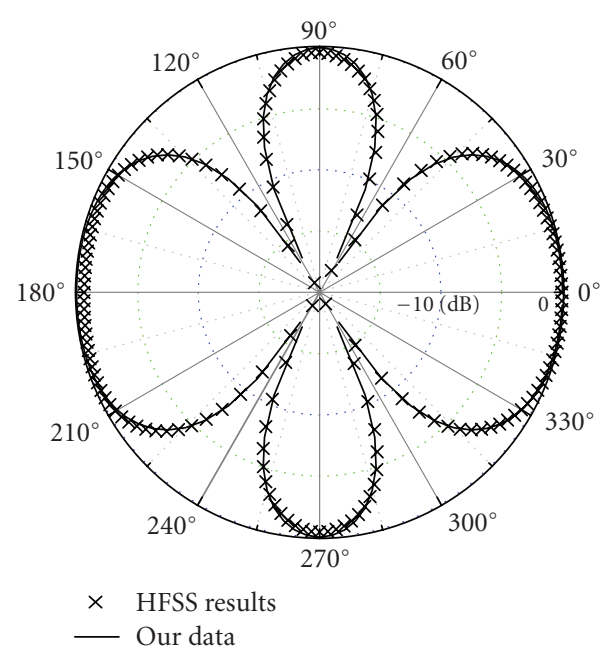

(b) $D=320 \mathrm{~mm}$

FIGURE 5: Comparison of our results for the two-monopole antenna's H-plane radiation pattern with those obtained by HFSS $(f=$ $\left.0.9375 \mathrm{GHz}, \theta=90^{\circ}, V_{1}=V_{2}=1 \mathrm{~V}\right)$.

well with those obtained by HFSS, which further validates our formulation. It should be pointed out that, in HFSS simulation, the monopole array is confined in a cylindrical region on the ground plane. The size of the ground plane must be a few-wavelength large to minimize the diffraction effect from its edge. The cylindrical region is bounded by absorbing conditions, which can simulate the outgoing waves in an open space.

In our modal-expansion analysis, a fictitious upper conducting boundary is introduced and the first-order approximation is employed for the electric currents flowing on the surfaces of monopole antennas. In order to examine the effect of the introduced boundary and the approximation made, a comparison between our modal-expansion results with HFSS data for the $S$-parameters of a two-monopole antenna is conducted for various distances between the two monopoles. It is seen from Figure 6 that they agree very well even when $D / \lambda_{0}$ is small. However, our modal-expansion method may deteriorate when electrically thick monopoles are closely spaced. For example, when $a / \lambda_{0}>0.1$ and the separation is as small as $D / \lambda_{0}=0.25$, the first-order approximation (axi-symmetrical current distribution) will not be good enough to represent the field variation along the monopole surfaces. 


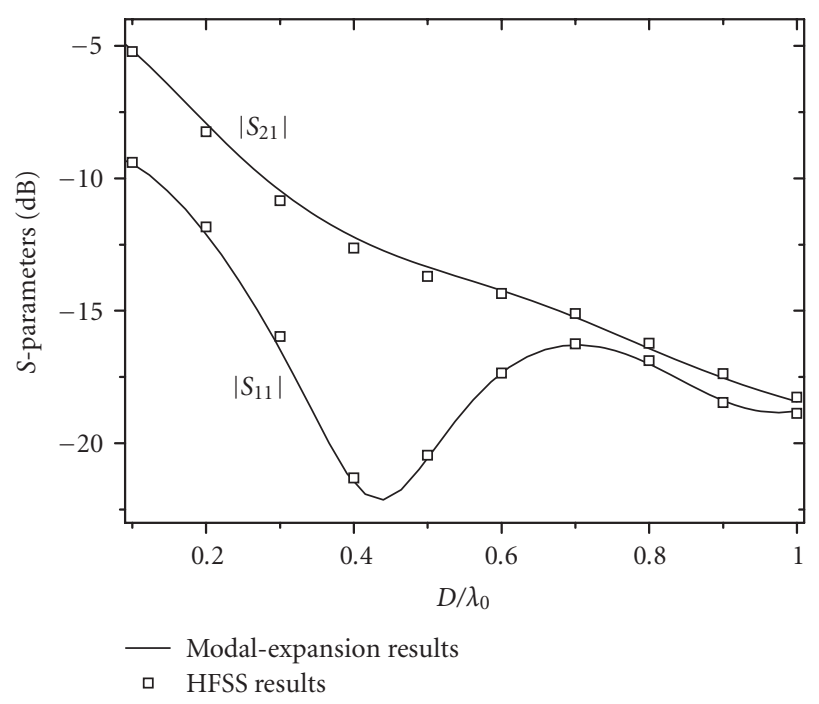

FIGURE 6: Variation of $S$-parameters of a two-monopole antenna with respect to their separation $(f=0.9375 \mathrm{GHz})$.

It should be mentioned that for a two-monopole antenna with a fixed $D$, the computation time to obtain its $S$ parameters for one frequency point using HFSS is $30 \mathrm{~min}-$ utes. However, it takes only 40 seconds for our modalexpansion method to get the $S$-parameters at the same frequency using the same computer $(2.8 \mathrm{GHz} \mathrm{CPU}$ and $512 \mathrm{M}$ RAM). This proves that our modal-expansion method is very computationally efficient.

As we have mentioned earlier, compared with the method of moments (MOM), one of the advantages that our modalexpansion method has is that it can be directly used to analyze thick monopoles, while the MOM with the thin-wire kernel is only applicable to thin monopoles. Figure 7 provides the comparison of our modal expansion results with MOM ones for the input impedance of the two-monopole antenna. It can be seen that they agree well when the monopole radius is electrically small. However, the MOM results deteriorate with the increase of the monopole radius. For a single frequency, the computation time for our modalexpansion method is about 20 seconds $\left(N_{1}=N_{2}=80\right)$, while for the MOM (mininec3 .exe is used, segments $=25$ are used for both monopoles), it is about 4 seconds on the same computer. Although the MOM is more efficient, our modalexpansion method can provide accurate results for both thin and thick monopoles.

Figure 8 shows the effect of the presence of another element on a monopole antenna's input admittance in the twomonopole array. Its relative parameters are the same as those in Figure 2. It is seen that the input conductance rises to $0.05 \mathrm{~S}$ from $0.03 S$ at the resonant frequency due to the mutual coupling between two monopoles. It is observed that the change in the input conductance is dependent upon the distance between two elements.

Figure 9 further examines the effect of the spacing between two monopoles on the mutual coupling. It can be seen that, with the increase of the spacing $D, Y_{11}$ approaches

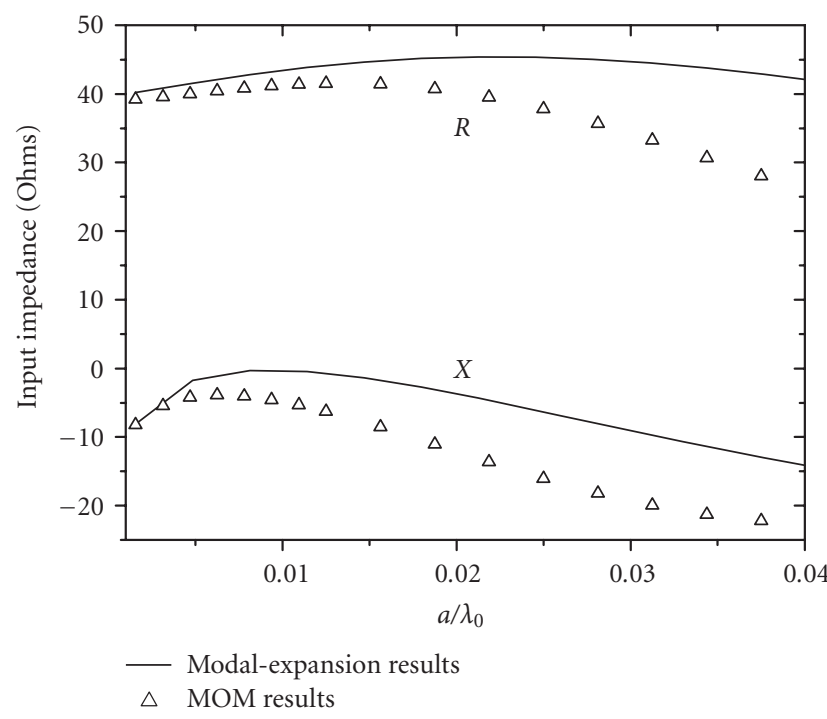

FIgURE 7: Variation of the input impedance of a two-monopole antenna with respect to their radii $(f=0.9375 \mathrm{GHz}, D=160 \mathrm{~mm}$ ).

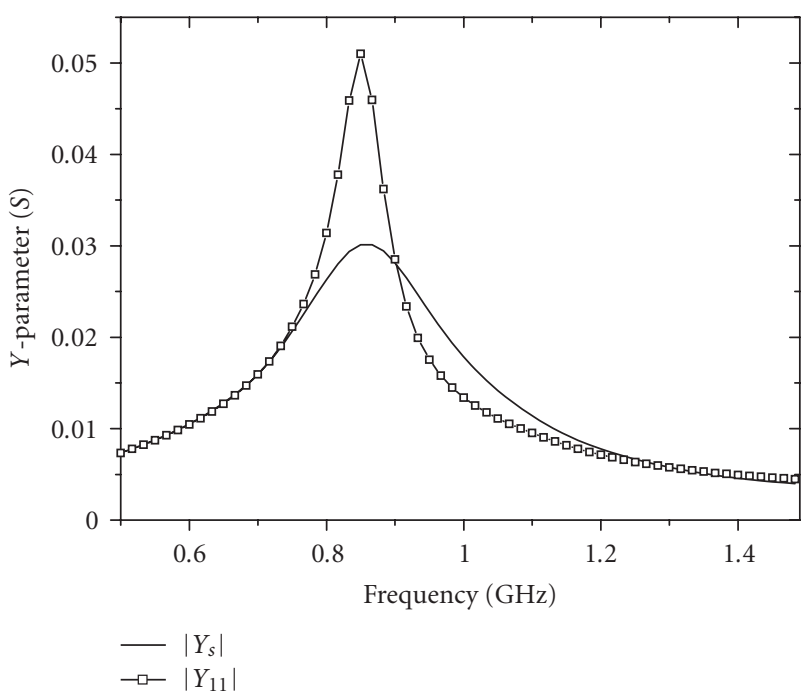

FIGURE 8: The effect of the mutual coupling between monopoles on the input admittance of a monopole antenna $(D=80 \mathrm{~mm})$.

a fixed value of $0.024 \mathrm{~S}$, which corresponds to the value of $Y_{s}$, also seen in Figure 8. However, $Y_{21}$ becomes smaller and smaller with the increase of the spacing due to the fact that the mutual coupling decreases with the increase of the spacing as expected.

As stated earlier, one advantage of our modal-expansion method is that it can be directly employed to analyze the mutual coupling between thick monopoles, unlike the moment method where the thin-wire kernel must be replaced with the thick-wire full-kernel for thick monopoles. As an example, a two-element array with thick monopoles was simulated using the modal-expansion method. Its relative parameters are given as $a_{1}=a_{2}=5.172 \mathrm{~mm}, h_{1}=h_{2}=12.57 \mathrm{~mm}, D=$ $25.86 \mathrm{~mm}$. The comparison between our modal-expansion 


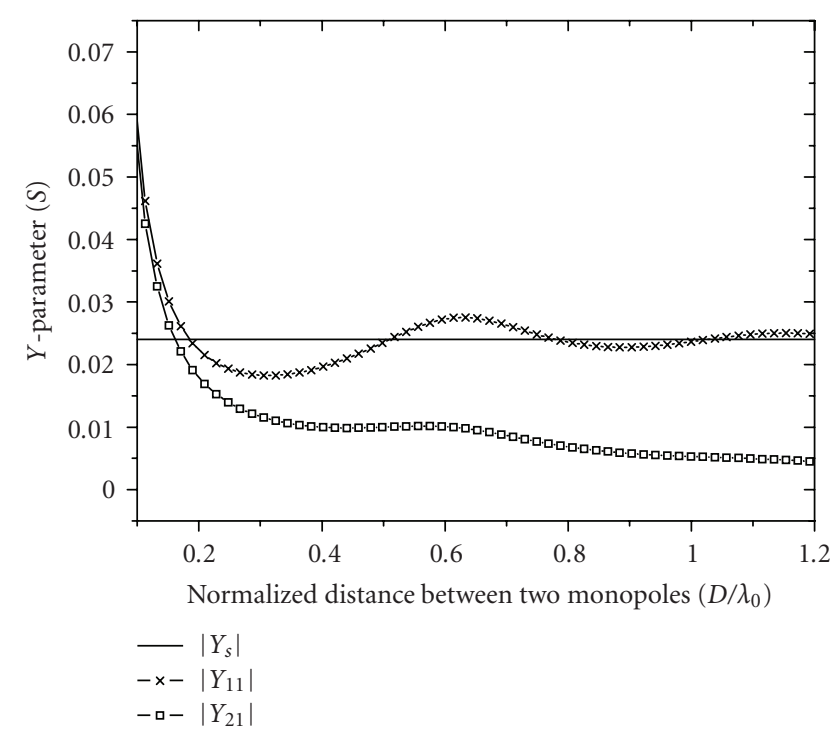

FIgURE 9: The effect of the monopole distance on the $Y$-parameters of the two-monopole antenna $(f=937.5 \mathrm{MHz})$.

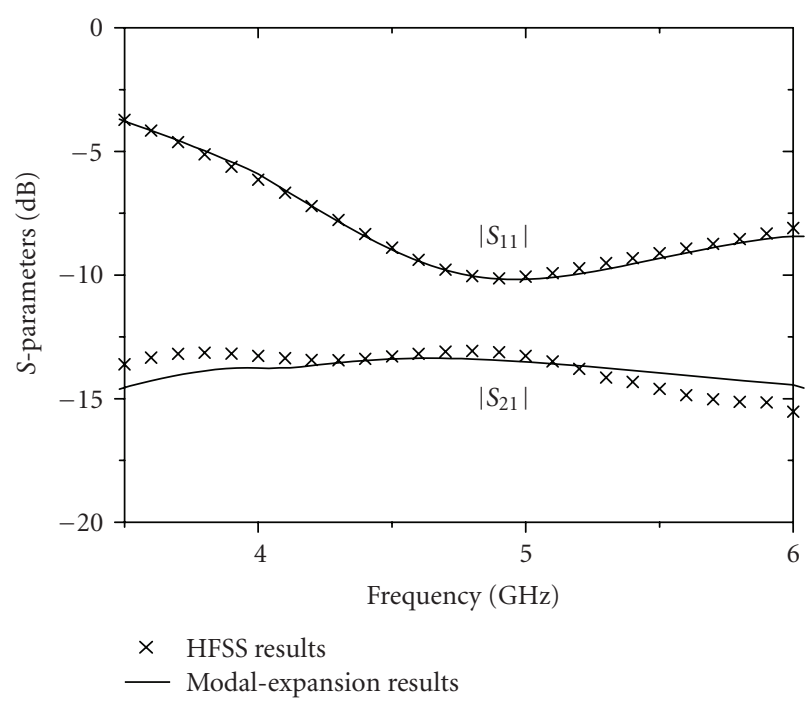

FIGURE 10: Simulated $S$-parameter of an array of two thick monopoles.

results and those obtained by HFSS for the $S$-parameters is good even when the monopole radius is almost $0.1 \lambda$, as shown in Figure 10.

As an example of a planar monopole array, a fourmonopole antenna capable of beam steering is considered, as shown in Figure 11. The four monopoles have identical length $h=29 \mathrm{~mm}$ and radius $a=0.8 \mathrm{~mm}$. The spacing between adjacent monopoles is fixed to be $D=61.26 \mathrm{~mm}$ or $D=122.45 \mathrm{~mm}$. The distance of the upper conducting plate and the ground plane is set to be $L=200 \mathrm{~mm}$ in our simulation.

Figure 12 presents the $S$-parameters of the four-monopole antenna when $D=61.26 \mathrm{~mm}$. The mutual coupling be-
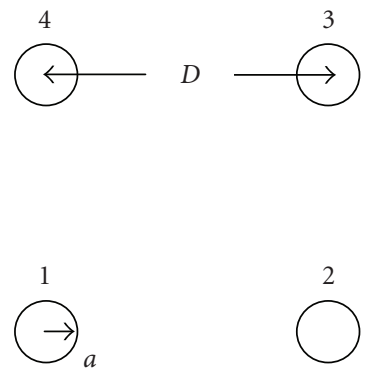

Figure 11: Top view of a four-monopole antenna $(a=0.8 \mathrm{~mm}$, $h=29 \mathrm{~mm}$ ).

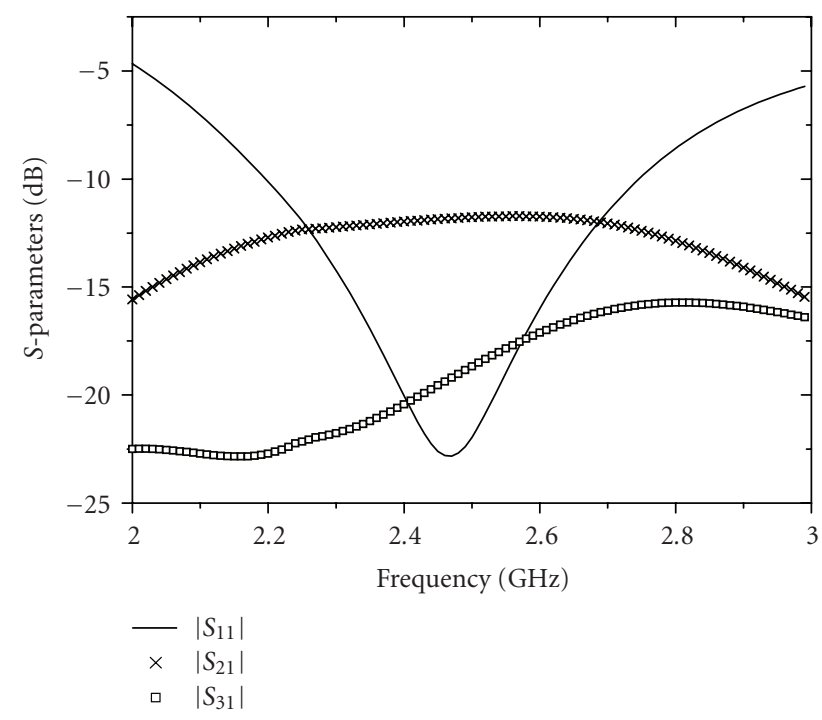

Figure 12: $S$-parameters of a four-monopole antenna $(D=$ $61.26 \mathrm{~mm})$.

tween monopoles can be easily seen from $s_{21}$ and $s_{31}\left(s_{41}=\right.$ $s_{21}$ ) in the figure. To verify our modal-expansion results, we compute the radiation patterns of the four-monopole antenna and compare them with simulated results from HFSS. Figure 13 shows the comparison of our modal-expansion results for the radiation patterns with those obtained by HFSS when $D=61.26 \mathrm{~mm}$ and $D=122.45 \mathrm{~mm}$, respectively. It is seen that the agreement between our results and HFSS data is good.

The beam-steering capability of a four-monopole antenna with $D=61.26 \mathrm{~mm}$ is examined in Figure 14. As expected, when $V_{1}=1 \mathrm{~V}, V_{2}=V_{3}=V_{4}=0$, the maximum directivity can be obtained at $225^{\circ}$. The radiation pattern can be steered by adjusting the magnitude of the excitations of the monopoles. When monopole 2 is fed with $V_{2}=5 \mathrm{~V}$, it is seen from Figure 14(b) that the maximum direction is changed to around $309^{\circ}$. Similarly, the radiation pattern can also be steered by adjusting the phases of the excitation sources of the monopoles. Figure 14(c) shows the radiation pattern of the four-monopole antenna when a source $V_{2}=e^{-j \pi / 2} \mathrm{~V}$ is applied to monopole 2 . We can see that the maximum direction is also changed. 


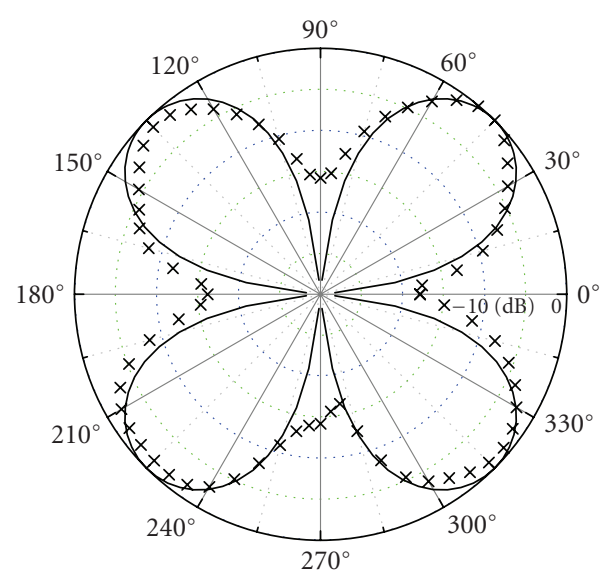

$\times \quad$ HFSS results

— Our data

(a) $D=61.26 \mathrm{~mm}$

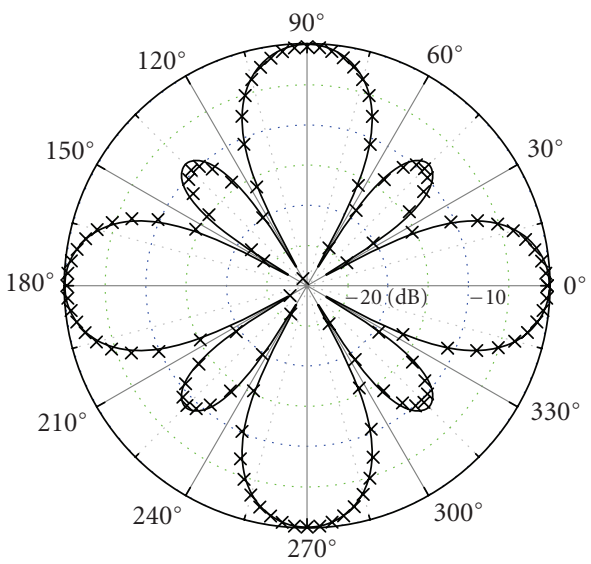

$\times$ HFSS results

— Our data

(b) $D=122.45 \mathrm{~mm}$

FIGURE 13: Comparison of our results with those obtained by HFSS for a four-monopole antenna's H-plane radiation pattern $(f=2.45 \mathrm{GHz}$, $V_{1}=V_{2}=V_{3}=V_{4}=1 \mathrm{~V}, \theta=90^{\circ}$.

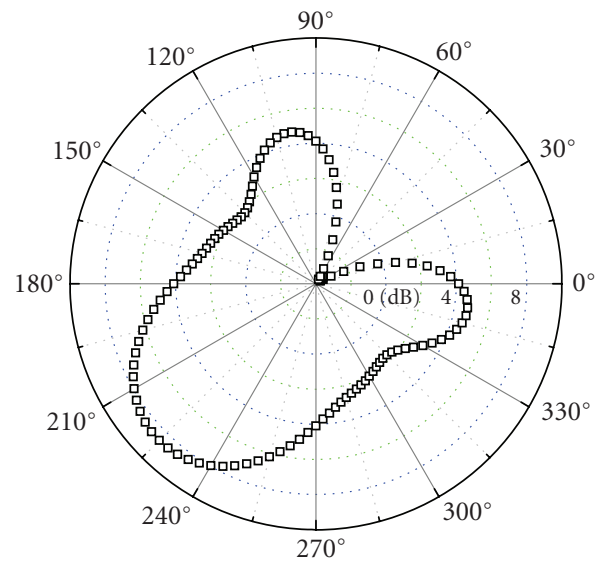

(a) $V_{1}=1 \mathrm{~V}, V_{2}=V_{3}=V_{4}=0$

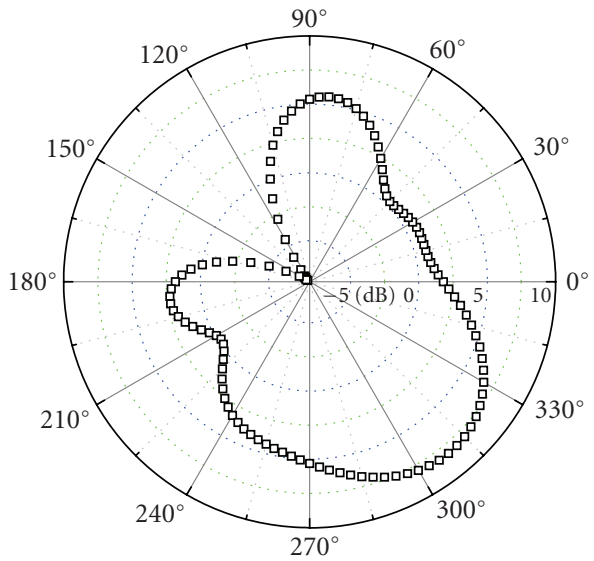

(b) $V_{1}=1 \mathrm{~V}, V_{2}=5 \mathrm{~V}, V_{3}=V_{4}=0$

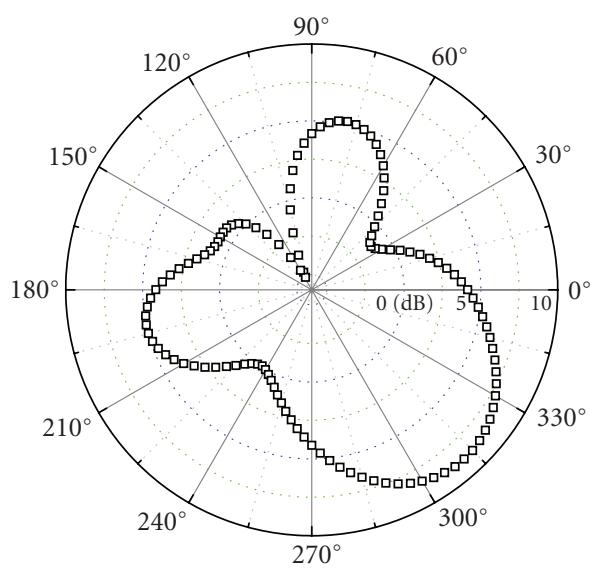

(c) $V_{1}=1 \mathrm{~V}, V_{2}=e^{-j \pi / 2} V, V_{3}=V_{4}=0$

FIGURE 14: H-plane gain patterns of a four-monopole antenna $\left(f=2.45 \mathrm{GHz}, D=61.26 \mathrm{~mm}, \theta=90^{\circ}\right)$. 


\section{CONCLUSION}

The radiation characteristics of an array of multiple monopole antennas driven by gap sources over an infinite ground plane have been studied in this paper. The modal-expansion method has been employed to analyze the antenna problem and cylindrical function's addition theorem has been used to facilitate the enforcement of boundary conditions. An experimental model of a twomonopole antenna has been constructed and measured results of its $S$-parameters are in good agreement with numerical ones. It has been demonstrated that the modal-expansion method can still give reasonably good results even when the monopoles are electrically thick and the spacing between them is as close as $0.1 \lambda_{0}$. The mutual coupling effect between two monopoles has been carefully examined. The radiation performance of a four-monopole antenna has also been studied. Simulated results have shown that it can be used to steer its radiation beam by properly adjusting its elements' excitation amplitudes and phases. Future extension of the problem considered will involve top-hat monopole arrays or monopole arrays coated with dielectric materials to enhance the directivity and improve the broadband matching.

\section{ACKNOWLEDGMENT}

The authors are very grateful to the anonymous reviewers for their valuable and constructive comments.

\section{REFERENCES}

[1] R. W. P. King, The Theory of Linear Antennas, Harvard University Press, Cambridge, Mass, USA, 1956.

[2] B. Tomasic and A. Hessel, "Linear array of coaxially fed monopole elements in a parallel plate waveguide-I: theory," IEEE Transactions on Antennas and Propagation, vol. 36, no. 4, pp. 449-462, 1988.

[3] D. C. Chang, "On the electrically thick monopole-part 1: theoretical solutions," IEEE Transactions on Antennas and Propagation, vol. 16, no. 1, pp. 58-64, 1968.

[4] M. E. Morris, "Analysis of a finite length tubular monopole antenna driven from a coaxial line," Tech. Rep. SAND79-1557, Sandia National Laboratories, Albuquerque, NM, USA, 1980.

[5] T. Do-Nhat and R. H. MacPhie, "The admittance of a monopole antenna fed through a ground plane by a coaxial line," IEEE Transactions on Antennas and Propagation, vol. 39, no. 8, pp. 1243-1247, 1991.

[6] C. D. Taylor, E. A. Aronson, and C. W. Harrison Jr., "Theory of coupled monopoles," IEEE Transactions on Antennas and Propagation, vol. 18, no. 3, pp. 360-366, 1970.

[7] R. F. Harrington, Field Computation by Moment Methods, IEEE Press, New York, NY, USA, 1993.

[8] J. Janapsatya and M. E. Bialkowski, "Reducing the height of a circular array of monopoles using top hats and dielectric coatings," Radio Science, vol. 39, no. 3, pp. 10 pages, 2004.

[9] H. Kawakami and T. Ohira, "Electrically steerable passive array radiator (ESPAR) antennas," IEEE Antennas and Propagation Magazine, vol. 47, no. 2, pp. 43-50, 2005.

[10] K. Gyoda and T. Ohira, "Design of electronically steerable passive array radiator (ESPAR) antennas," in IEEE Antennas and
Propagation Society International Symposium (APS '00), vol. 2, pp. 922-925, Salt Lake City, Utah, USA, July 2000.

[11] C. Sun, A. Hirata, T. Ohira, and N. C. Karmakar, "Fast beamforming of electronically steerable parasitic array radiator antennas: theory and experiment," IEEE Transactions on Antennas and Propagation, vol. 52, no. 7, pp. 1819-1832, 2004.

[12] F. Schwering, N. Puri, and A. Stavridis, "The modal expansion solution of a top loaded dipole," in IEEE Antennas and Propagation Society International Symposium (APS '82), vol. 2, pp. 584-587, Albuquerque, NM, USA, May 1982.

[13] M. A. Morgan, R. C. Hurley, and F. K. Schwering, "Computation of monopole antenna currents using cylindrical harmonics," IEEE Transactions on Antennas and Propagation, vol. 38, no. 7, pp. 1130-1133, 1990.

[14] M. A. Morgan and F. K. Schwering, "Eigenmode analysis of dielectric loaded top-hat monopole antennas," IEEE Transactions on Antennas and Propagation, vol. 42, no. 1, pp. 54-61, 1994.

[15] Z. Shen and R. H. MacPhie, "Modal expansion analysis of monopole antennas driven from a coaxial line," Radio Science, vol. 31, no. 5, pp. 1037-1046, 1996.

[16] Z. Shen and R. H. MacPhie, "Rigorous evaluation of the input impedance of a sleeve monopole by modal-expansion method," IEEE Transactions on Antennas and Propagation, vol. 44, no. 12, pp. 1584-1591, 1996.

[17] M. E. Bialkowski, F. K. Schwering, and M. A. Morgan, "On the link between top-hat monopole antennas, disk-resonator diode mounts, and coaxial-to-waveguide transitions," IEEE Transactions on Antennas and Propagation, vol. 48, no. 6, pp. 1011-1014, 2000.

[18] M. E. Bialkowski and P. W. Davis, "Analysis of a circular patch antenna radiating in a parallel-plate radial guide," IEEE Transactions on Antennas and Propagation, vol. 50, no. 2, pp. 180187, 2002.

[19] Z. N. Chen, K. Hirasawa, and K. Wu, "A novel top-sleeve monopole in two parallel plates," IEEE Transactions on Antennas and Propagation, vol. 49, no. 3, pp. 438-443, 2001.

[20] M. Abramowitz and I. A. Stegun, Handbook of Mathematical Functions, Dover, New York, NY, USA, 1970.

[21] M. M. Weiner, "Monopole element at the center of a circular ground plane whose radius is small or comparable to a wavelength," IEEE Transactions on Antennas and Propagation, vol. 35, no. 5, pp. 488-495, 1987.

\section{AUTHOR CONTACT INFORMATION}

Quanxin Wang: Division of Communication Engineering, School of Electrical and Electronic Engineering, Nanyang Technological University, 50 Nanyang Avenue, Singapore 639798; qxwang.assoc@ntu.edu.sg

Zhongxiang Shen: Division of Communication Engineering, School of Electrical and Electronic Engineering, Nanyang Technological University, 50 Nanyang Avenue, Singapore 639798; ezxshen@ntu.edu.sg

Erping Li: Computational Electromagnetics and Electronics Division, Institute of High Performance Computing, National University of Singapore, 1 Science Park Road, Singapore 117528; elelep@nus.edu.sg 

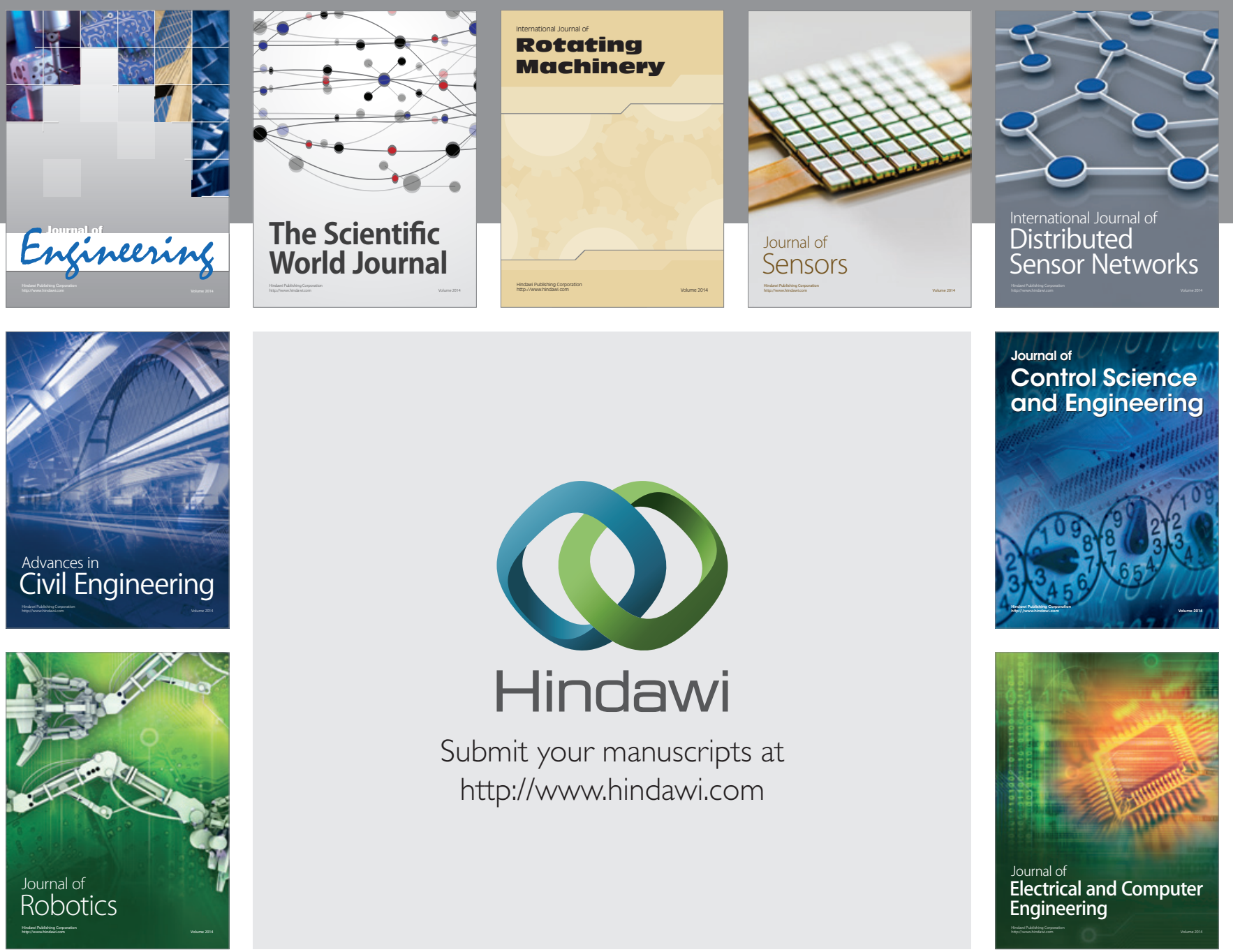

Submit your manuscripts at

http://www.hindawi.com
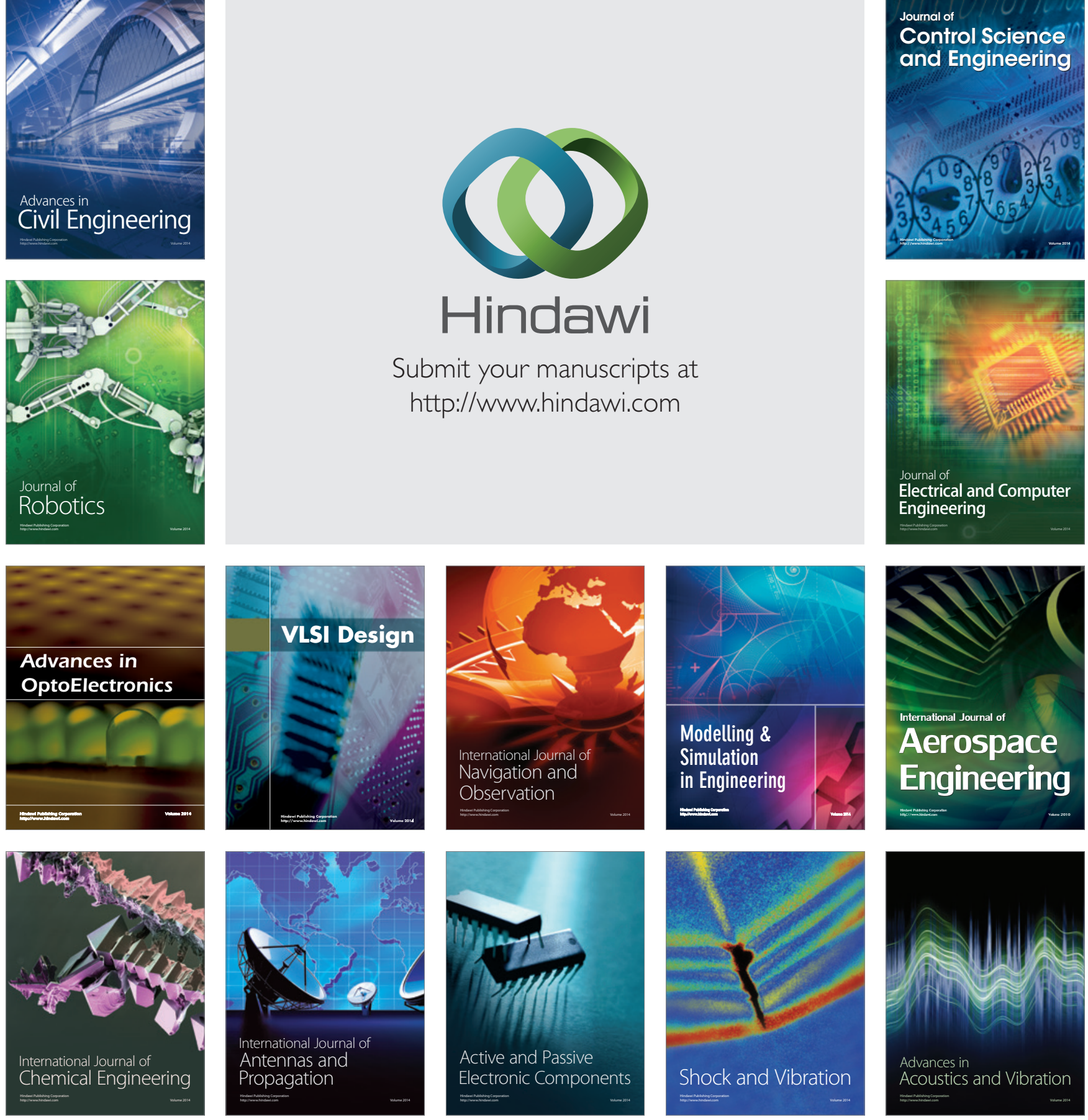